\title{
3 Research Square

\section{Visualization of human optic nerve and tract by diffusion tensor mapping and degree of neuropathy}

\section{Łukasz Łabieniec}

Department of Condensed Matter Physics, University of Bialystok

tukasz Lisowski

Department of Ophthalmology, Medical University of Bialystok

Horia Petrache

Department of Physics, Indiana University Purdue University Indianapolis

Marcin Hładuński

Independent Laboratory of Molecular Imaging, Medical University of Bialystok

Joanna Konopińska ( $\sim$ joannakonopinska@o2.pl )

Department of Ophthalmology, Medical University of Bialystok

Jan Kochanowicz

Department of Neurology, Medical University of Bialystok

Krzysztof Szymański

Department of Condensed Matter Physics, University of Bialystok

\section{Research Article}

Keywords: diffusion MRI, diffusion tensor, DTI, optic nerve, optic tract, visual pathway, optic nerve atrophy, neuropathy, tensor mapping, degree of neuropathy

Posted Date: February 15th, 2022

DOI: https://doi.org/10.21203/rs.3.rs-1322662/v1

License: (c) (i) This work is licensed under a Creative Commons Attribution 4.0 International License.

Read Full License 


\section{Abstract}

Diffusion-weighted magnetic resonance imaging of the human optic nerve and tract is technically difficult because of its small size, the inherent strong signal generated by the surrounding fat and the cerebrospinal fluid, and due to eddy current-induced distortions and subject movement artifacts. In addition, the effects of the bone channel through which the optic nerve passes, and the proximity of blood vessels, muscles and tendons are generally unknown. Furthermore, the limited technical capabilities of the scanners and the minimization of acquisition times result in poor quality diffusionweighted images. It is challenging for current tractography methods to accurately track optic pathway fibers that correspond to known anatomy and to produce a reasonable fraction of decussating fibers. Despite these technical limitations and low image resolution, here we show how to visualize the optic nerve and tract and quantify nerve atrophy. Our visualization method based on the analysis of the diffusion tensor shows marked differences between a healthy male subject and a male subject with progressive optic nerve neuropathy, differences that are otherwise not visible on standard morphological images or scalar diffusion maps. Importantly, our method provides a quantification of the degree of optic nerve atrophy in a systematic way.

\section{Highlights}

Standard MRI morphological images fail to show the functionality of the optic nerve. Current limitations and artifacts due to eddy currents and susceptibility effects on scan acquisition together with unavoidable patient movement put serious limits on the quality of diffusion weighted images. Standard manual and semi-automatic segmentations, as well as tractography, do not always reproduce the well know anatomy surrounding the optic nerve. Here we show a data analysis algorithm and a procedure to accurately assess the degree of the optic nerve atrophy based only on actual diffusion tensor data.

\section{Introduction}

In biological tissues, water translational diffusion is influenced by microstructural components, including cell membranes and organelles. The optic nerve is an almost pure white matter tract that has an intrinsically high water restricted diffusion due to its highly packed axons and the encompassing nerve sheaths [1]. By adjusting acquisition parameters, the movement of water molecules can be measured by magnetic resonance imaging (MRI). The most advanced MRI technique proposed in 1994 [2] allows the measurement of directional diffusion and is called diffusion tensor imaging (DTI) [3-7]. This method comprises a group of techniques in which the eigenvalues and eigenvectors of the diffusion tensor are used to create images reflecting various diffusion properties of a tissue [8-10]. DTI can provide information on anatomical connectivity in the brain by measuring the anisotropic diffusion of water in white matter tracts [11-20] or in gray matter structures [21]. One of the most commonly used measures is fractional anisotropy $(F A)$, which quantifies how strong directional diffusion in the local voxel is. Many imaging studies are starting to use $F A$ images from multiple subjects in voxelwise tract-based spatial statistical analyses, in order to localize brain changes related to development, training, degeneration or 
disease, and to explore anatomical connectivity in the brain and compare with other nonhuman primates [22-27]. In this method, called tractography, "seed voxels" are selected in a certain area of the brain, and then fiber trajectories are computed by an automated software.

Tractography is a method for identifying white matter pathways in the living human brain and it is the only available tool for non-invasive and in vivo identification and measurement of these pathways. Fiber trajectories form the substrate for information transfer between remote brain regions and are therefore central to understanding of function in both the normal and diseased brain. Although many studies have shown tractography to be of promising value for neurosurgical care $[28,29]$, some results remain inconclusive [30]. The idea of fiber tracking is to follow the primary eigenvector from the selected seed voxel (or group of voxels) to encounter neighboring voxels, at which the trajectory is changed according to the direction of the new eigenvector in the neighboring voxel. Fiber tracking ends when one of the following conditions is met: 1 ) the tract reaches the boundary of the imaging volume, 2) the tract reaches a region with low diffusion anisotropy, 3 ) the radius of curvature of the tract is smaller than a selected threshold radius, or 4) the most collinear eigenvector is not associated with the largest eigenvalue [31]. The resulting trajectories can be used as a region of interest for $F A$ and other scalar parameters voxelbased measurements.

In principle, the DTI technique should be helpful in visualizing the optic nerve and tract. Unfortunately, because of the insufficient spatial resolution of the images, the optic nerve, as well as spinal cord, are technically difficult to investigate with any MRI technique, especially DTI [32]. It is challenging for current tractography methods to accurately track optic pathway fibers that correspond to known anatomy and reproduce reasonably decussating fibers. However, systematic progress is being reported [33] and several studies have shown successful tracking of some parts of the optic pathway [34-38]. Examples of visualization of retinogeniculate visual pathway fiber displacement due to tumors have been reported in $[36,37,39]$.

The optic nerve is surrounded by cerebrospinal fluid and fat, which significantly affect the measured signal. It is also not known what the effect of the presence of nearby bony structures, veins, or even muscles and tendons is (Fig. 1). An abnormal signal near the optic canal (Fig. 1c), where the optic nerve is surrounded by bone and air, results in negative diffusion tensor eigenvalues [33]. The small size of the optic nerve and its movement during scanning make it even harder to obtain high-quality images. Additionally, sequences such as planar imaging (EPI) used in DTI are more sensitive to image artifacts and eddy current distortions than any other MRI technique [40-42]. Limitations of the EPI include low spatial resolution, blurring effects of T2 decay occurring during image readout, and sensitivity to artifacts due to Nyquist ghosting, chemical shift, magnetic field inhomogeneity, and local susceptibility effects [43, 44]. Although it is possible to obtain a high-resolution tractography of the optic pathway in animals, the acquisition time is several dozen hours [45] or even few days [46]. Owing to the technical limitations of the current scanners and long acquisition time requirements, it is impossible to obtain good-quality diffusion images of the optic pathway and hence probabilistic tractography results. Optic pathway shape and anatomy make this problem even more demanding. All the way to the back of the brain requires 
going through the lateral geniculate nucleus and turning off the fiber near Myer's loop. This procedure is very challenging because the curvature of the optic nerve is very high, and thus, the DTI-based method tracking loses the optic nerves at that point. Through the optic chiasm fibers cross in some cases and merge or branch, going in and out on the same side. None of the tractography methods are inherently able to properly deal with this complicated topology and with the intricate morphology of crossing and branching. Scalar diffusion parameters of the optic nerve are much more often measured by manual selection of the area of interest by qualified radiologists or from tracts found with tractography, manually trimmed by an experienced physician to better match the anatomy [47].

Several studies have found strong correlations between the severity of glaucoma and a reduction in $F A$, as well as an increase in mean diffusivity $(M D)$ in the optic nerve [48-51]. Patients with optic neuritis have been found to have increased $M D$, and decreased $F A$ [52], with similar findings reported in patients with multiple sclerosis [53]. Higher $M D$ have been found in retinitis pigmentosa than in healthy controls, as well as lower $F A$ [54]. The amblyopic patients showed significant reductions in optic nerve and optic tract microstructural integrity with decreasing $F A$ in the optic nerve and optic tract [47]. However, because of the low resolution of the diffusion images, the results are often inconclusive, and correlations are not sufficiently strong. Because of this, is it difficult to assess the exact degree of the optic nerve functionality such as in visual evoked potentials measurements [55]. In some cases [56], for example in optic neuritis [57] or glaucoma [58], the optic nerve can be assessed from morphological images based on T1 or T2.

In the standard DTI visualization, the principal component of the diffusion tensor at each voxel is displayed with line segments [59]. It is also possible to display multiple line segments per voxel that show the second and the third eigenvectors. For color coding of displayed line segments, standard RGB convention is used where the colors red, green and blue represent diffusion along the $x, y$-and $z$-axes, respectively. Three eigenvectors can also be visualized in the form of an ellipsoid with symmetry axes parallel to the eigenvectors and the color depending on the dominating eigenvalue $[4,7,60]$. With our algorithm, we significantly improve the visibility of the optic nerve, chiasm and tract by an analysis of eigenvectors and eigenvalues and an optimized assignment of visual components that include segments length, opacity and color depth which together enhance the visibility of the fiber structures.

\section{Materials And Methods}

\section{Study design}

In the present study, images from a healthy male subject (age: 32 ) and a male subject with penetrating right eyeball injury (age: 46 ) were used. Both subjects underwent a full ophthalmological examination,

which included visual acuity, color vision, applanation measurement of intraocular pressure, evaluation of the anterior chamber and the fundus of the eye using a slit lamp and the Volk lens, optical coherence tomography examination of the macula and optic nerve disc (OCT), neurological field vision, and visual evoked potential (VEP) testing. In the tests conducted, no deviations from the normal condition were found in the subjects, while the trauma patient had endotamponade of the vitreous chamber of the right 
eye with silicone oil, lack of sense of light, secondary glaucoma, and atrophy of the right eye optic nerve, and correct ophthalmological examination of the left eye. In his medical history, the patient reported that prior to the penetrating injury (metal shard from a masonry hammer driven into the eyeball), the patient did not experience any deterioration in vision compared to the left eye. During active scanning, the patients tried not to blink and did not move their heads, while gazing at the red-locking LED outside the camera reflected in the mirror of the coil.

\section{Diffusion tensor imaging}

MRI data were acquired using a Biograph mMR 3-Tesla scanner (Siemens, Erlangen, Germany) with a 16channel head-neck coil. Acquisition parameters were set up based on the article [61] and technician experience. The total duration of the EPI DTI examination was 10:34 min (8:05 and 2:29 for A >>P and $\mathrm{P} \gg>\mathrm{A}$ phase encoding directions respectively). MRI acquisitions were obtained using a scanner with a maximum gradient amplitude of $45 \mathrm{mT} / \mathrm{m}$ and a maximum slew rate of $200 \mathrm{~T} / \mathrm{m} / \mathrm{s}$. Parameters for used for echo planar (EPI) acquisition method were: TR $7000 \mathrm{~ms}$, TE $113 \mathrm{~ms}$, echo spacing $0.97 \mathrm{~ms}$, bandwidth $1184 \mathrm{~Hz} / \mathrm{Px}, \mathrm{EPI}$ factor 128, voxel size 1.3×1.3×2.0 mm, 25 slices (transverse orientation), distance factor $0 \%$ for transverse plane and $50 \%$ for other planes, base resolution 128 and prescan normalization filtering. Sixty-four non-collinear diffusion directions for a $b$ value of $1000 \mathrm{~s} / \mathrm{mm}^{2}$ with one average were acquired for the $A \gg>P$ encoding direction and six averages without diffusion-weighted acquisition for $b$ value of $0 \mathrm{~s} / \mathrm{mm}^{2}$ were acquired for both the $A \gg P$ and the $P \gg>A$ encoding directions. Parallel imaging with an acceleration factor of 2 was enabled using the GRAPPA algorithm. Additionally, an anatomical T1-weighted (MP RAGE), scan time 5:21 min, voxel size 1.0×1.0×1.2 mm, was performed.

In addition to coronal magnetization-prepared rapid acquisition gradient-echo reconstruction, oblique transverse sections through both optic nerves were done. The exact positions and angulation of slices were further graphically specified and adjusted in the central sections of the left and right sagittal optic nerves. This procedure allowed for an exact adjustment of the EPI and T1 slices through both optic nerves. In general, positioning was focused on the part of the optic nerve closest to the eye to allow for optimal comparability in the case of a curved or kinked optic nerve.

\section{Image post-processing}

Diffusion images were converted from the DICOM format to the NIfTI format using the open source dcm2niix software [62], and the data were post-processed using the FSL software library (http://www.fmrib.ox.ac.uk/fsl) [63]. The ITK-SNAP software (http://www.itksnap.org) was used to create a binary mask of the brain, eyeballs, and optic nerves. FSL's tools were used for susceptibility -induced and eddy current -induced distortion correction, and movement artifacts correction [64-66]. The correction procedure assumes that the eddy current-induced field can be modelled as a combination of linear and quadratic terms. Calculations were parallelized with CUDA v9.1 on Nvidia Tesla K80 GPU. Computations were carried out at the Computer Center of University of Bialystok, Poland. Eddy current and motion-corrected diffusion-weighted images were used for voxel-by-voxel-based tensor calculations. Consequently, eigenvectors $\left(V_{1}, V_{2}\right.$ and $\left.V_{3}\right)$ and eigenvalues $\left(L_{1} \geq L_{2} \geq L_{3}\right)$ were obtained. 
Throughout the text, the largest eigenvalue $L_{1}$ will be called axial diffusivity. For all other calculations, the Wolfram Mathematica software was used and NIfTI images were imported using the QMRITools toolbox developed for the Wolfram language [67]. Fractional anisotropy $(F A)$, mean diffusivity $(M D)$ and radial diffusivity $(R D)$ were defined as $[7,43,61]$ :

$F A=\frac{\left(L_{1}-L_{2}\right)^{2}+\left(L_{2}-L_{3}\right)^{2}+\left(L_{1}-L_{3}\right)^{2}}{2\left(L_{1}^{1}+L_{2}^{2}+L_{3}^{2}\right)}$,

$M D=\left(L_{1}+L_{2}+L_{3}\right) / 3$

$R D=\left(L_{2}+L_{3}\right) / 2$

\section{Algorithm}

The physical property of the diffusion tensor is that all eigenvalues are non-negative. However, correction algorithms and low-resolution data can lead to negative eigenvalues that are clearly non-physical. Therefore, in our procedure all negative, nonphysical eigenvalues were set to zero [68]. The data visualization algorithm consisted of six steps each having a significant impact on the displayed image. The visual effect accomplished by each step is presented in Fig. 2a-f and described below.

In the first step, the first eigenvector $V_{1}$ in each voxel is displayed as a line segment representing the main direction of diffusion in that voxel, and the line segments are given the same length (Fig. 2a). Because the morphological structure of the optic nerve favors the presence of one particularly distinguished direction of diffusion, we should focus especially on voxels in which diffusion is strongly directional i.e., $L_{1} \gg L_{2}$. Hence, in the second step, the length of the line segment in each voxel is adjusted to a value given by

$\alpha\left(L_{1}-R D\right)^{2}$

where $\alpha$ is a scaling parameter. This means that the more $L_{1}$ deviates from $R D$, the longer the line segment for that voxel (Fig. 2b).

Another important piece of information is the strength of diffusion in each voxel. Thus, in the third step, the opacity of the line segment in each voxel is set proportional to the product of the mean and radial diffusivities,

$\beta(M D F A)$, (5)

where $\beta$ is a scaling parameter. This means that the stronger the diffusion, the more opaque the segment for that voxel (Fig. 2c). 
The 4th step in our algorithm is an RGB color assignment that improves image clarity based on $L_{1}$ values as follows

$\operatorname{Red}=\gamma L_{1 N}$

Green $=1-L_{1 N}$

Blue $=0$, (6)

where $\gamma$ is a scaling parameter for setting the intensity of red color, and $L_{1 N}$ is a normalized value, $L_{1 N}=L_{1} / L_{1 \max ^{\prime}}$ where $L_{1 \max }$ is the maximal value among all voxels $L_{1}$ (Fig. 2 d).

The goal of the standard tractography color coding rejecting is to assume that the $L_{1}$ values should be similar along the entire length of the optic pathway, and therefore, it should be presented in a similar color. Furthermore, standard tractography color coding appears to be ineffective for the optic pathway because this structure does not form a straight-line segment in three-dimensional projections and such coding would unnecessarily introduce different colors on different sections of the optic nerve and tract. For this reason, for optic pathway visualization, more than one layer must be used. Therefore, we propose a new approach. In step five, we projected the three-dimensional shape of the visual pathways onto the transverse plane (Fig. 2e). Consequently, although the information about shape in the direction of the projection is lost, we gain a much-improved visibility in the two other directions (i.e., the plane perpendicular to the projection direction). On the transverse plane, the visual pathway is best visualized, but to show the complete location of the nerve, we project manually the segmented optic pathway onto the sagittal and coronal planes (Fig. 3). After the projection of a few layers onto one plane, we obtain a superposition of line segments in each voxel, producing enhanced visibility. However, segment superposition can affect image clarity and for this reason we implement a 6th step in which short line segments are discarded based on the following cutoff condition

$L_{1}>R D+\sigma$,

where $\sigma$ is a threshold value (Fig. 5). The result is that line segments are displayed only if the axial diffusivity exceeds the radial diffusivity by more than a chosen value for $\sigma$ (Fig. 2f).

The presented algorithm and projections in three directions are particularly suited for the imaging of fiber structures. The optic nerves are clearly visualized in Fig. 3 (arrows). The right-hand side nerve is visualized as a single fiber, while the left-hand side nerve is split on coronal and sagittal projections because it is located just between the two planes used in the imaging protocol. Because the imaging protocol includes pre-scan normalization filtering, the parameters $\alpha, \beta, \gamma$ and $\sigma$ were selected once to ensure the best visibility of the visual pathways. These parameters may require modifications if any changes in the protocol arise or when different scanner devices are used. An unexpected but useful result is that the parameter $\sigma$ can serve as a quantitative measure in the diagnostics of nerve atrophy as shown later in the manuscript. 


\section{Image quality testing}

Diffusion tensor estimation can produce indefinite diffusion tensors that arise in locations where the diffusion-weighted signal is disturbed by noise and the degree of anisotropy is high [69]. In biological tissue, all eigenvalues of the diffusion tensor are assumed to be positive but due to noise or signal drop, negative eigenvalues may be generated [68]. The fraction of voxels for which negative eigenvalues have been generated relative to the total number of all voxels, expressed as a percentage, can be used as a

measure of image quality. For a healthy subject, those values were $0.61 \%, 6.5 \%$ and $9.0 \%$ for $L_{1}, L_{2}$ and $L_{3}$ maps, respectively. In the case of a subject with a damaged eyeball, these values reached the values of $0.99 \%, 2.3 \%$, and $9.5 \%$, respectively.

\section{Results}

The presented algorithm was used to generate images of the visual pathway in a healthy male subject (Fig. 4b) and a male subject with the right optic nerve atrophy caused by eyeball injury (Fig. 4a). Progressive optic neuropathy in the second subject showed no changes in the T1 image and little, insignificant changes in FA and $M D$ scalar diffusion maps (Fig. 4). In contrast, the DTI image represented as line segments generated by the algorithm presented in this work showed marked difference between the atrophic and fully functional optic nerves.

A set of images generated for different values of $\sigma$ show that this parameter can be used as an indicator of the degree of neuropathy (Fig. 5). For the healthy male subject, the optic nerves disappear from the image when $\sigma$ exceeds 0.0013 and 0.0014 for the left and right optic nerves, respectively. In contrast, for the injured male subject, the atrophic nerve is not visible even for $\sigma$ equal to zero (Fig. 5a) while the left, healthy optic nerve disappears for a $\sigma$ value of 0.0014 (Fig. 5c).

It is important to note that the presented algorithm allows visualization of the oculomotor nerve (indicated by the white arrow in Fig. 2d), consistent with the anatomical atlases [70-72]. The bone channel through which the optic nerve passes (optic channel) may be the reason for the observed optic nerve discontinuities at this point (marked with blue arrows in Fig. 4).

\section{Discussion}

Visualization of the optic nerve is necessary because in many cases where patients report visual impairment, changes are seen on electroencephalography but not on MRI images. The electroencephalographic result itself is sometimes ambiguous and in order to make an accurate diagnosis, it is necessary to compare it with another method. Apart from clinical and electrophysiological evaluation, MRI plays an important role in the complete assessment of optic nerve and the entire visual pathway. MRI images are helpful in describing the segmental anatomy of the optic nerve and to findings of various conditions affecting the optic nerves [56]. Because of the insufficient spatial resolution of the diffusion MRI images, it is difficult to assess the functionality of the optic nerve. 
The presented algorithm showed the possibility of a direct visualization of data for the optic nerve by diffusion tensor mapping, without relying on probabilistic imaging tools. We have shown that appropriate matching and combining scalar values of the diffusion tensor with the eigenvector data can result in the generation of an image that shows a significant advantage in functional assessment compared to single scalar maps or morphological images. Although some slight differences for atrophic and healthy optic nerves can be seen on the $F A$ and $M D$ maps, the atrophic and healthy nerves are better differentiated with our algorithm.

We have shown that the projection of a few layers on a single plane is a preferential method to view fibrous tissues. Even though some 3D information is lost, the visibility enhancement of the entire structure is significant. Since multilayer projections cannot be merged with single layer morphological images due to possible mismatch, in Fig. 4 we show only a part of the T1 image in the background.

In diffusion tensor imaging, the subject uncontrolled blinking, eyeball movements, and spatial and temporal variations of the static magnetic field caused by susceptibility effects and time-varying eddy currents result in severe distortions, blurring, and misregistration artifacts [64-66, 73]. Due to the limitations of acquisition time and attempts to shorten it to a minimum for the patient's comfort, only the part of the head containing the optic nerve and the tract was scanned.

It is important for our protocol that the orientation of layers is chosen such that they are parallel to the optic nerve axis to a large extent, which is different than standard brain scanning. We tested our algorithm on some publicly available sets of diffusion images. For example, the Human Connectome Project's team has released diffusion imaging and structural imaging data acquired from 35 young adults using a customized MGH Siemens 3T Connectome scanner [74], which has a maximum gradient strength of $300 \mathrm{mT} / \mathrm{m}$ for diffusion imaging, which is up to an order of magnitude more powerful than standard clinical scanners. Moreover, the section planes were oblique to the optic nerve axis.

By finding the $\sigma$ value at which the optic nerve disappears from the image we obtain a quantitative measure of optic nerve functionality. Other numerical voxel-based measurements are possible by optic nerve segmentation from images in which voxel brightness intensities are set based on the product of segment length and opacity. In such a monochrome image, it is easy to draw a region of interest's mask, for example, on the part of the optic nerve starting behind the eyeball and ending on the skull optic canal, where the line continuity is lost. Founded masks can be automatically corrected by $\sigma$ parameter, which may be a threshold value used for semi-automatic segmentation [75], or active contour segmentation [76]. Both methods can be alternatives to different tractography segmentations [33] and manual methods.

\section{Conclusions}

The proposed new approach of diffusion tensor visualization of the optic nerve and tract is a better method than the standard tractography methods, which often produces results that do not fit well with the known anatomy. Our method properly accounts for fiber curvature without relying on probabilistic 
imaging tools. The images generated by the algorithm presented in this study clearly show significant differences between the atrophic and healthy optic nerves from the eyeball to the optic chiasm and the sigma parameter in our algorithm provides a quantitative measure of nerve atrophy.

\section{Declarations}

\section{Data availability}

The schematic anatomical overview of optic pathway supporting Fig. 1 and the NIfTI images supporting Figs. $2-5$, are publicly available in the figshare repository, as part of this record: https://doi.org/10.6084/m9.figshare.19076660.v1 [77].

\section{Acknowledgements}

The authors gratefully acknowledge to Dr. Peter Basser and the members of his Section on Quantitative Imaging and Tissue Sciences lab from the National Institute of Health (Bethesda, USA) for their helpful comments and suggestions.

The authors gratefully acknowledge to Kamil Cepuch from Siemens Healthcare GmbH, Erlangen, Germany, for his invaluable advice.

The authors gratefully acknowledge the access to the scanner by BioSkaner, Bialystok, Poland and thanks Dr. Malgorzata Mojsak for institutional help.

L.L. ackwnowleges the support of the Department of Physics at Indiana University Purdue University during his Fulbright Fellowship.

The research work was financed from the Polish Ministry of Science. Computations were carried out at the Computer Center of University of Bialystok, Poland.

\section{Ethics statement}

This study was performed in compliance with the Declaration of Helsinki and was approved by the local ethics commission (Medical University of Bialystok under the number R-I-002/477/2019). Written and informed consent was obtained from all subjects prior to study inclusion.

\section{References}

1. He, M.; Cestari, D.; Cunnane, M.B.; Rizzo, J.F. The Use of Diffusion MRI in Ischemic Optic Neuropathy and Optic Neuritis. Seminars in Ophthalmology 2010, 25, 225-232, doi:10.3109/08820538.2010.518450. 
2. Basser, P.J.; Mattiello, J.; Lebihan, D. Estimation of the Effective Self-Diffusion Tensor from the NMR Spin Echo. Journal of Magnetic Resonance, Series B 1994, 103, 247-254, doi:10.1006/jmrb.1994.1037.

3. Basser, P.J.; Mattiello, J.; LeBihan, D. MR Diffusion Tensor Spectroscopy and Imaging. Biophysical journal 1994, 66, 259-267, doi:10.1016/S0006-3495(94)80775-1.

4. Basser, P.J. Inferring Microstructural Features and the Physiological State of Tissues from Diffusion Weighted Images. NMR in BIOMEDICINE 1995, 8, 333-344.

5. Tournier, J.-D.; Mori, S.; Leemans, A.; Morgan, R.H.; Reson, M.; Author, M. Diffusion Tensor Imaging and Beyond NIH Public Access Author Manuscript. Magn Reson Med 2011, 65, 1532-1556, doi:10.1002/mrm.22924.Diffusion.

6. Mukherjee, P.; Berman, J.I.; Chung, S.W.; Hess, C.P.; Henry, R.G. Diffusion Tensor MR Imaging and Fiber Tractography: Theoretic Underpinnings., doi:10.3174/ajnr.A1051.

7. Minati, L.; Węglarz, W.P. Physical Foundations, Models, and Methods of Diffusion Magnetic Resonance Imaging of the Brain: A Review. Concepts Magn Reson Part A 2007, 30, 278-307, doi:10.1002/cmr.a.20094.

8. Kingsley, P.B. Introduction to Diffusion Tensor Imaging Mathematics: Part I. Tensors, Rotations, and Eigenvectors. Concepts in Magnetic Resonance Part A: Bridging Education and Research 2006, 28 , 101-122, doi:10.1002/cmr.a.20048.

9. Kingsley, P.B. Introduction to Diffusion Tensor Imaging Mathematics: Part II. Anisotropy, DiffusionWeighting Factors, and Gradient Encoding Schemes. Concepts in Magnetic Resonance Part A: Bridging Education and Research 2006, 28, 123-154, doi:10.1002/cmr.a.20049.

10. Kingsley, P.B. Introduction to Diffusion Tensor Imaging Mathematics: Part III. Tensor Calculation, Noise, Simulations, and Optimization. Concepts in Magnetic Resonance Part A: Bridging Education and Research 2006, 28, 155-179, doi:10.1002/cmr.a.20050.

11. Wu, W.; Miller, K.L. Image Formation in Diffusion MRI: A Review of Recent Technical Developments. Journal of Magnetic Resonance Imaging 2017, 46, 646-662, doi:10.1002/jmri.25664.

12. Lazar, M.; Alexander, A.L. Bootstrap White Matter Tractography (BOOT-TRAC). Neurolmage 2005, 24, 524-532, doi:10.1016/J.NEUROIMAGE.2004.08.050.

13. Lazar, M.; Weinstein, D.M.; Tsuruda, J.S.; Hasan, K.M.; Arfanakis, K.; Meyerand, M.E.; Badie, B.; Rowley, H.A.; Haughton, V.; Field, A.; et al. White Matter Tractography Using Diffusion Tensor Deflection. Human Brain Mapping 2003, 18, 306-321, doi:10.1002/HBM.10102.

14. McIntosh, A.M.; Maniega, S.M.; Lymer, G.K.S.; McKirdy, J.; Hall, J.; Sussmann, J.E.D.; Bastin, M.E.; Clayden, J.D.; Johnstone, E.C.; Lawrie, S.M. White Matter Tractography in Bipolar Disorder and Schizophrenia. Biological Psychiatry 2008, 64, 1088-1092, doi:10.1016/J.BIOPSYCH.2008.07.026.

15. Lazar, M. Mapping Brain Anatomical Connectivity Using White Matter Tractography. NMR in Biomedicine 2010, 23, 821-835, doi:10.1002/NBM.1579.

16. Chen, X.; Weigel, D.; Ganslandt, O.; Buchfelder, M.; Nimsky, C. Diffusion Tensor Imaging and White Matter Tractography in Patients with Brainstem Lesions. Acta Neurochirurgica 2007 149:11 2007, 
149, 1117-1131, doi:10.1007/S00701-007-1282-2.

17. Moody, J.F.; Adluru, N.; Alexander, A.L.; Field, A.S. The Connectomes: Methods of White Matter Tractography and Contributions of Resting State FMRI. Seminars in Ultrasound, CT and MRI 2021, 42, 507-522, doi:10.1053/J.SULT.2021.07.007.

18. Deng, L.; Sui, R.; Zhang, L. Diffusion Tensor Tractography Characteristics of White Matter Tracts Are Associated with Post-Stroke Depression. Neuropsychiatric Disease and Treatment 2021, 17, 167, doi:10.2147/NDT.S274632.

19. Lerch, J.P.; van der Kouwe, A.J.W.; Raznahan, A.; Paus, T.; Johansen-Berg, H.; Miller, K.L.; Smith, S.M.; Fischl, B.; Sotiropoulos, S.N. Studying Neuroanatomy Using MRI. Nature Neuroscience 2017 20:3 2017, 20,314-326, doi:10.1038/nn.4501.

20. Mollink, J.; Smith, S.M.; Elliott, L.T.; Kleinnijenhuis, M.; Hiemstra, M.; Alfaro-Almagro, F.; Marchini, J.; van Cappellen van Walsum, A.M.; Jbabdi, S.; Miller, K.L. The Spatial Correspondence and Genetic Influence of Interhemispheric Connectivity with White Matter Microstructure. Nature Neuroscience 2019 22:5 2019, 22, 809-819, doi:10.1038/s41593-019-0379-2.

21. Behrens, T.E.J.; Johansen-Berg, H.; Woolrich, M.W.; Smith, S.M.; Wheeler-Kingshott, C.A.M.; Boulby, P.A.; Barker, G.J.; Sillery, E.L.; Sheehan, K.; Ciccarelli, O.; et al. Non-Invasive Mapping of Connections between Human Thalamus and Cortex Using Diffusion Imaging. Nature Neuroscience 2003 6:7 2003, 6, 750-757, doi:10.1038/nn1075.

22. Smith, S.M. Fast Robust Automated Brain Extraction. Human Brain Mapping 2002, 17, 143-155, doi:10.1002/hbm.10062.

23. Smith, S.M.; Jenkinson, M.; Woolrich, M.W.; Beckmann, C.F.; Behrens, T.E.J.; Johansen-Berg, H.; Bannister, P.R.; de Luca, M.; Drobnjak, I.; Flitney, D.E.; et al. Advances in Functional and Structural MR Image Analysis and Implementation as FSL. In Proceedings of the Neurolmage; 2004; Vol. 23.

24. Smith, S.M.; Jenkinson, M.; Johansen-Berg, H.; Rueckert, D.; Nichols, T.E.; Mackay, C.E.; Watkins, K.E.; Ciccarelli, O.; Cader, M.Z.; Matthews, P.M.; et al. Tract-Based Spatial Statistics: Voxelwise Analysis of Multi-Subject Diffusion Data. Neurolmage 2006, 31, 1487-1505, doi:10.1016/j.neuroimage.2006.02.024.

25. Scholz, J.; Klein, M.C.; Behrens, T.E.J.; Johansen-Berg, H. Training Induces Changes in White-Matter Architecture. Nature Neuroscience 2009 12:11 2009, 12, 1370-1371, doi:10.1038/nn.2412.

26. Bengtsson, S.L.; Nagy, Z.; Skare, S.; Forsman, L.; Forssberg, H.; Ullén, F. Extensive Piano Practicing Has Regionally Specific Effects on White Matter Development. Nature Neuroscience 2005 8:9 2005, 8, 1148-1150, doi:10.1038/nn1516.

27. Rilling, J.K.; Glasser, M.F.; Preuss, T.M.; Ma, X.; Zhao, T.; Hu, X.; Behrens, T.E.J. The Evolution of the Arcuate Fasciculus Revealed with Comparative DTI. Nature Neuroscience 2008 11:4 2008, 11, 426428, doi:10.1038/nn2072.

28. Fukui, M.B.; Monroy-Sosa, A.; Chakravarthi, S.S.; Jennings, J.E.; Rovin, R.A.; Epping, A.; Kassam, A.B. Integration of White Matter Tractography in Subcortical and Skull Base Neurosurgical Planning. Principles of Neuro-Oncology 2021, 245-264, doi:10.1007/978-3-030-54879-7_11. 
29. Essayed, W.I.; Zhang, F.; Unadkat, P.; Cosgrove, G.R.; Golby, A.J.; O’Donnell, L.J. White Matter Tractography for Neurosurgical Planning: A Topography-Based Review of the Current State of the Art. Neurolmage: Clinical 2017, 15, 659-672, doi:10.1016/J.NICL.2017.06.011.

30. Wende, T.; Hoffmann, K.T.; Meixensberger, J. Tractography in Neurosurgery: A Systematic Review of Current Applications. Journal of neurological surgery. Part A, Central European neurosurgery 2020, 81, 442-455, doi:10.1055/S-0039-1691823.

31. Basser, P.J.; Pajevic, S.; Pierpaoli, C.; Duda, J.; Aldroubi, A. In Vivo Fiber Tractography Using DT-MRI Data. Magnetic Resonance in Medicine 2000, 44, 625-632, doi:10.1002/1522-2594.

32. Barker, G.J. Diffusion-Weighted Imaging of the Spinal Cord and Optic Nerve. Journal of the Neurological Sciences 2001, 186, 45-49, doi:10.1016/S0022-510X(01)00490-7.

33. He, J.; Zhang, F.; Xie, G.; Yao, S.; Feng, Y.; Bastos, D.C.A.; Rathi, Y.; Makris, N.; Kikinis, R.; Golby, A.J.; et al. Comparison of Multiple Tractography Methods for Reconstruction of the Retinogeniculate Visual Pathway Using Diffusion MRI. Human Brain Mapping 2021, 42, 3887-3904, doi:10.1002/HBM.25472.

34. Altıntaş, Ö.; Gümüştaş, S.; Cinik, R.; Anık, Y.; Özkan, B.; Karabaş, L. Correlation of the Measurements of Optical Coherence Tomography and Diffuse Tension Imaging of Optic Pathways in Amblyopia. International ophthalmology 2017, 37, 85-93, doi:10.1007/S10792-016-0229-0.

35. Hofer, S.; Karaus, A.; Frahm, J. Reconstruction and Dissection of the Entire Human Visual Pathway Using Diffusion Tensor MRI. Frontiers in neuroanatomy 2010, 4, 1-7, doi:10.3389/FNANA.2010.00015.

36. Lober, R.M.; Guzman, R.; Cheshier, S.H.; Fredrick, D.R.; Edwards, M.S.B.; Yeom, K.W. Application of Diffusion Tensor Tractography in Pediatric Optic Pathway Glioma. Journal of neurosurgery. Pediatrics 2012, 10, 273-280, doi:10.3171/2012.7.PEDS1270.

37. Panesar, S.S.; Abhinav, K.; Yeh, F.C.; Jacquesson, T.; Collins, M.; Fernandez-Miranda, J. Tractography for Surgical Neuro-Oncology Planning: Towards a Gold Standard. Neurotherapeutics: the journal of the American Society for Experimental NeuroTherapeutics 2019, 16, 36-51, doi:10.1007/S13311018-00697-X.

38. Yoshino, M.; Abhinav, K.; Yeh, F.C.; Panesar, S.; Fernandes, D.; Pathak, S.; Gardner, P.A.; FernandezMiranda, J.C. Visualization of Cranial Nerves Using High-Definition Fiber Tractography. Neurosurgery 2016, 79, 146-165, doi:10.1227/NEU.0000000000001241.

39. Potgieser, A.R.E.; Wagemakers, M.; van Hulzen, A.L.J.; de Jong, B.M.; Hoving, E.W.; Groen, R.J.M. The Role of Diffusion Tensor Imaging in Brain Tumor Surgery: A Review of the Literature. Clinical neurology and neurosurgery 2014, 124, 51-58, doi:10.1016/J.CLINEURO.2014.06.009.

40. Bodammer, N.; Kaufmann, J.; Kanowski, M.; Tempelmann, C. Eddy Current Correction in DiffusionWeighted Imaging Using Pairs of Images Acquired with Opposite Diffusion Gradient Polarity. Magnetic Resonance in Medicine 2004, 51, 188-193, doi:10.1002/mrm.10690.

41. Reese, T.G.; Heid, O.; Weisskoff, R.M.; Wedeen, V.J. Reduction of Eddy-Current-Induced Distortion in Diffusion MRI Using a Twice-Refocused Spin Echo. Magnetic Resonance in Medicine 2003, 49, 177- 
182, doi:10.1002/mrm.10308.

42. Bastin, M.E. Correction of Eddy Current-Induced Artefacts in Diffusion Tensor Imaging Using Iterative Cross-Correlation. Magnetic Resonance Imaging 1999, 17, 1011-1024, doi:10.1016/S0730725X(99)00026-0.

43. Mukherjee, P.; Chung, S.W.; Berman, J.I.; Hess, C.P.; Henry, R.G. Diffusion Tensor MR Imaging and Fiber Tractography: Technical Considerations. PHYSICS REVIEW AJNR Am J Neuroradiol 2008, 29, 843-852, doi:10.3174/ajnr.A1052.

44. Mukherjee, P.; Berman, J.I.; Chung, S.W.; Hess, C.P.; Henry, R.G. Diffusion Tensor MR Imaging and Fiber Tractography: Theoretic Underpinnings., doi:10.3174/ajnr.A1051.

45. Wedeen, V.J.; Wang, R.P.; Schmahmann, J.D.; Benner, T.; Tseng, W.Y.I.; Dai, G.; Pandya, D.N.; Hagmann, P.; D’Arceuil, H.; de Crespigny, A.J. Diffusion Spectrum Magnetic Resonance Imaging (DSI) Tractography of Crossing Fibers. Neurolmage 2008, 41, 1267-1277, doi:10.1016/J.NEUROIMAGE.2008.03.036.

46. Liu, C.; Ye, F.Q.; Newman, J.D.; Szczupak, D.; Tian, X.; Yen, C.C.C.; Majka, P.; Glen, D.; Rosa, M.G.P.; Leopold, D.A.; et al. A Resource for the Detailed 3D Mapping of White Matter Pathways in the Marmoset Brain. Nature Neuroscience 2020 23:2 2020, 23, 271-280, doi:10.1038/s41593-019-05750 .

47. Allen, B.; Schmitt, M.A.; Kushner, B.J.; Rokers, B. Retinothalamic White Matter Abnormalities in Amblyopia. Investigative Ophthalmology \& Visual Science 2018, 59, 921-929, doi:10.1167/IOVS.1722930.

48. Zhang, Q.J.; Wang, D.; Bai, Z.L.; Ren, B.C.; Li, X.H. Diffusion Tensor Imaging of Optic Nerve and Optic Radiation in Primary Chronic Angle-Closure Glaucoma Using 3T Magnetic Resonance Imaging. International Journal of Ophthalmology 2015, 8, 975, doi:10.3980/J.ISSN.2222-3959.2015.05.22.

49. Wang, M.Y.; Wu, K.; Xu, J.M.; Dai, J.; Qin, W.; Liu, J.; Tian, J.; Shi, D. Quantitative 3-T Diffusion Tensor Imaging in Detecting Optic Nerve Degeneration in Patients with Glaucoma: Association with Retinal Nerve Fiber Layer Thickness and Clinical Severity. Neuroradiology 2013, 55, 493-498, doi:10.1007/S00234-013-1133-1.

50. Li, K.; Lu, C.; Huang, Y.; Yuan, L.; Zeng, D.; Wu, K. Alteration of Fractional Anisotropy and Mean Diffusivity in Glaucoma: Novel Results of a Meta-Analysis of Diffusion Tensor Imaging Studies. PLOS ONE 2014, 9, doi:10.1371/journal.pone.0097445.

51. Chen, Z.; Lin, F.; Wang, J.; Li, Z.; Dai, H.; Mu, K.; Ge, J.; Zhang, H. Diffusion Tensor Magnetic Resonance Imaging Reveals Visual Pathway Damage That Correlates with Clinical Severity in Glaucoma. Clinical \& Experimental Ophthalmology 2013, 41, 43-49, doi:10.1111/J.14429071.2012.02832.X.

52. Trip, S.A.; Wheeler-Kingshott, C.; Jones, S.J.; Li, W.Y.; Barker, G.J.; Thompson, A.J.; Plant, G.T.; Miller, D.H. Optic Nerve Diffusion Tensor Imaging in Optic Neuritis. Neurolmage 2006, 30, 498-505, doi:10.1016/J.NEUROIMAGE.2005.09.024. 
53. RS, S.; M, K.; DL, T.; MR, S.; P, C.; DH, M.; CA, W.-K. Development of a High-Resolution Fat and CSFSuppressed Optic Nerve DTI Protocol at 3T: Application in Multiple Sclerosis. Functional neurology 2013, 28, doi:10.11138/FNEUR/2013.28.2.093.

54. Zhang, Y.; Guo, X.; Wang, M.; Wang, L.; Tian, Q.; Zheng, D.; Shi, D. Reduced Field-of-View Diffusion Tensor Imaging of the Optic Nerve in Retinitis Pigmentosa at 3T. AJNR: American Journal of Neuroradiology 2016, 37, 1510, doi:10.3174/AJNR.A4767.

55. Odom, J.V.; Bach, M.; Brigell, M.; Holder, G.E.; McCulloch, D.L.; Mizota, A.; Tormene, A.P. ISCEV Standard for Clinical Visual Evoked Potentials: (2016 Update). Documenta Ophthalmologica 2016 133:1 2016, 133, 1-9, doi:10.1007/S10633-016-9553-Y.

56. Gala, F. Magnetic Resonance Imaging of Optic Nerve. The Indian Journal of Radiology \& Imaging 2015, 25, 421, doi:10.4103/0971-3026.169462.

57. Rizzo, J.F.; Andreoli, C.M.; Rabinov, J.D. Use of Magnetic Resonance Imaging to Differentiate Optic Neuritis and Nonarteritic Anterior Ischemic Optic Neuropathy. Ophthalmology 2002, 109, 1679-1684, doi:10.1016/S0161-6420(02)01148-X.

58. Stroman, G.A.; Stewart, W.C.; Golnik, K.C.; Curé, J.K.; Olinger, R.E. Magnetic Resonance Imaging in Patients With Low-Tension Glaucoma. Archives of Ophthalmology 1995, 113, 168-172, doi:10.1001/ARCHOPHT.1995.01100020050027.

59. Tournier, J.D.; Mori, S.; Leemans, A. Diffusion Tensor Imaging and Beyond. Magnetic Resonance in Medicine 2011, 65, 1532, doi:10.1002/MRM.22924.

60. Mathys, C.; Aissa, J.; Zu Hörste, G.M.; Reichelt, D.C.; Antoch, G.; Turowski, B.; Hartung, H.P.; Sheikh, K.A.; Lehmann, H.C. Peripheral Neuropathy: Assessment of Proximal Nerve Integrity By Diffusion Tensor Imaging. Muscle \& Nerve 2013, 48, 889-896, doi:10.1002/MUS.23855.

61. Gerlach, D.A.; Marshall-Goebel, K.; Hasan, K.M.; Kramer, L.A.; Alperin, N.; Rittweger, J. MRI-Derived Diffusion Parameters in the Human Optic Nerve and Its Surrounding Sheath during Head-down Tilt. npj Microgravity 2017, 3, 18, doi:10.1038/s41526-017-0023-y.

62. Li, X.; Morgan, P.S.; Ashburner, J.; Smith, J.; Rorden, C. The First Step for Neuroimaging Data Analysis: DICOM to NIfTI Conversion. Journal of Neuroscience Methods 2016, 264, 47-56, doi:10.1016/j.jneumeth.2016.03.001.

63. Jenkinson, M.; Beckmann, C.F.; Behrens, T.E.; Woolrich, M.W.; Smith, S.M. FSL; 2011;

64. Andersson, J.L.R.; Sotiropoulos, S.N. An Integrated Approach to Correction for Off-Resonance Effects and Subject Movement in Diffusion MR Imaging. Neurolmage 2016, 125, 1063-1078, doi:10.1016/j.neuroimage.2015.10.019.

65. Andersson, J.L.R.; Sotiropoulos, S.N. Non-Parametric Representation and Prediction of Single- and Multi-Shell Diffusion-Weighted MRI Data Using Gaussian Processes. Neurolmage 2015, 122, 166 176, doi:10.1016/j.neuroimage.2015.07.067.

66. Graham, M.S.; Drobnjak, I.; Jenkinson, M.; Zhang, H. Quantitative Assessment of the Susceptibility Artefact and Its Interaction with Motion in Diffusion MRI. PLOS ONE 2017, 12, doi:10.1371/journal.pone.0185647. 
67. Froeling, M. QMRTools: A Mathematica Toolbox for Quantitative MRI Analysis. Journal of Open Source Software 2019, 4, 1204, doi:10.21105/JOSS.01204.

68. Batchelor, P.G.; Moakher, M.; Atkinson, D.; Calamante, F.; Connelly, A. A Rigorous Framework for Diffusion Tensor Calculus. Magnetic resonance in medicine 2005, 53, 221-225, doi:10.1002/MRM.20334.

69. Niethammer, M.; Estepar, R.S.J.; Bouix, S.; Shenton, M.; Westin, C.F. On Diffusion Tensor Estimation. Conference proceedings: ... Annual International Conference of the IEEE Engineering in Medicine and Biology Society. IEEE Engineering in Medicine and Biology Society. Conference 2006, 1, 2622, doi:10.1109/IEMBS.2006.259826.

70. Šimić, G. Heines DE. Neuroanatomy Atlas in Clinical Context: Structures, Sections, Systems, and Syndromes: 10th Edition; Baltimore: Wolters Kluwer (Lippincott Williams Wilkins); 2019. 384 Pages; ISBN 978-1-975106-68-3, EISBN: 978-1-4963-8416-4. Croatian Medical Journal 2019, 60, 390, doi:10.3325/CMJ.2019.60.390.

71. Cranial Nerves: Anatomy, Pathology, Imaging. https://doi.org/10.1148/radiol.11114010 2011, 260, 41-41, doi:10.1148/RADIOL.11114010.

72. DeArmond, S.J.; Fusco, M.M.; Dewey, M.M. Structure of the Human Brain: A Photographic Atlas. 1976, 186.

73. Truong, T.K.; Chen, N. kuei; Song, A.W. Dynamic Correction of Artifacts Due to Susceptibility Effects and Time-Varying Eddy Currents in Diffusion Tensor Imaging. Neurolmage 2011, 57, 1343, doi:10.1016/J.NEUROIMAGE.2011.06.008.

74. Fan, Q.; Witzel, T.; Nummenmaa, A.; van Dijk, K.R.A.; van Horn, J.D.; Drews, M.K.; Somerville, L.H.; Sheridan, M.A.; Santillana, R.M.; Snyder, J.; et al. MGH-USC Human Connectome Project Datasets with Ultra-High b-Value Diffusion MRI. Neurolmage 2016, 124, 1108-1114, doi:10.1016/J.NEUROIMAGE.2015.08.075.

75. Yushkevich, P.A.; Gao, Y.; Gerig, G. ITK-SNAP: An Interactive Tool for Semi-Automatic Segmentation of Multi-Modality Biomedical Images. Conference proceedings: ... Annual International Conference of the IEEE Engineering in Medicine and Biology Society. IEEE Engineering in Medicine and Biology Society. Annual Conference 2016, 2016, 3342, doi:10.1109/EMBC.2016.7591443.

76. Yushkevich, P.A.; Piven, J.; Hazlett, H.C.; Smith, R.G.; Ho, S.; Gee, J.C.; Gerig, G. User-Guided 3D Active Contour Segmentation of Anatomical Structures: Significantly Improved Efficiency and Reliability. Neurolmage 2006, 31, 1116-1128, doi:10.1016/j.neuroimage.2006.01.015.

77. Labieniec, L. NIfTI_images.Zip. 2022, doi:10.6084/m9.figshare.19076660.v1.

\section{Figures}




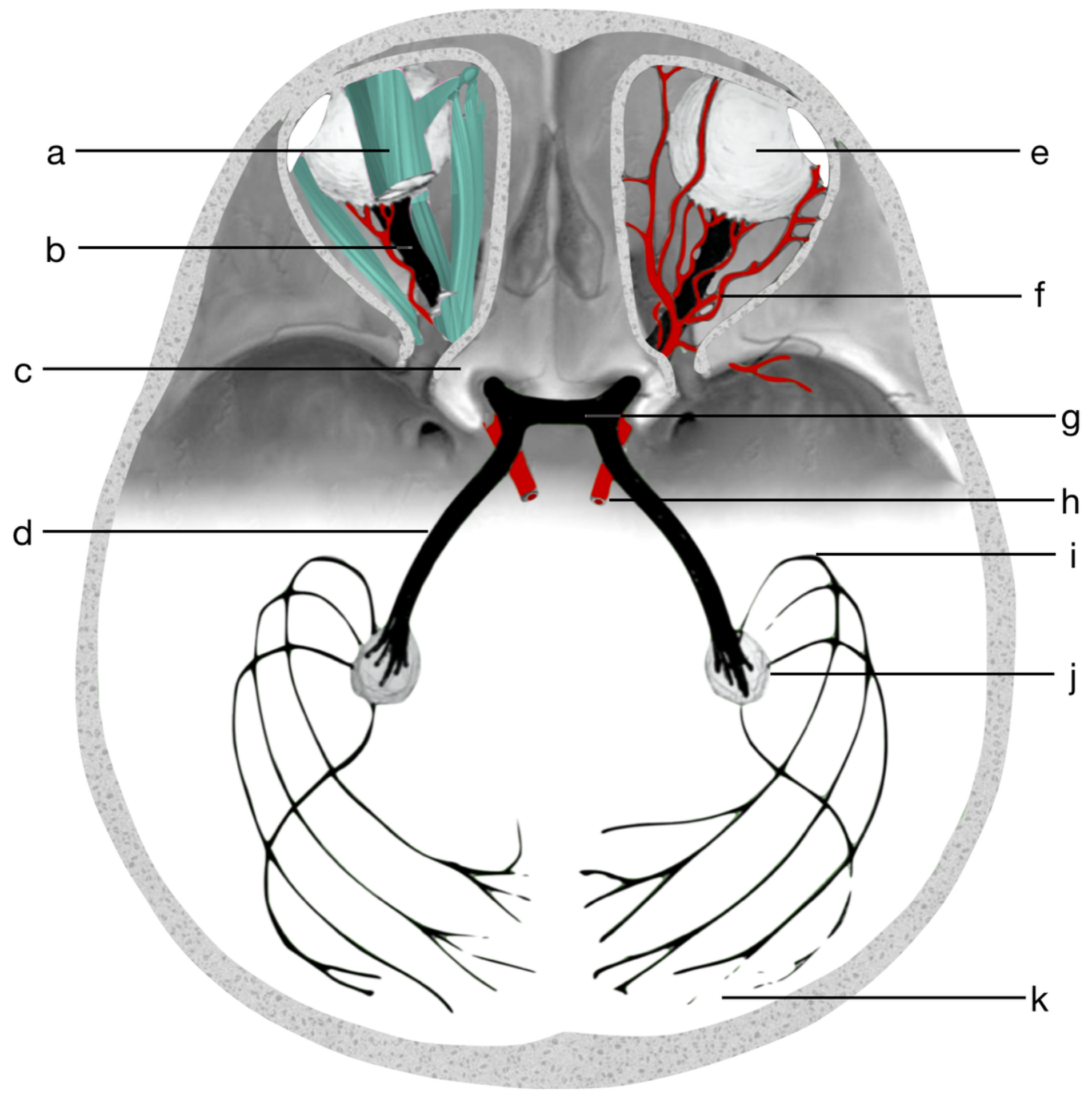

Figure 1

A schematic anatomical overview of optic pathway and surroundings, a - muscles and tendons, b optic nerve, c - nearby bony structures (optic canal), $d$ - optic tract, $\mathrm{e}$ - eyeball, $\mathrm{f}$ - blood vessels, $\mathrm{g}$ optic chiasm, h - ophthalmic artery, i - Meyer's loop, $\mathrm{j}$ - lateral geniculate nucleus, $\mathrm{k}$ - optic radiation and visual cortex. 


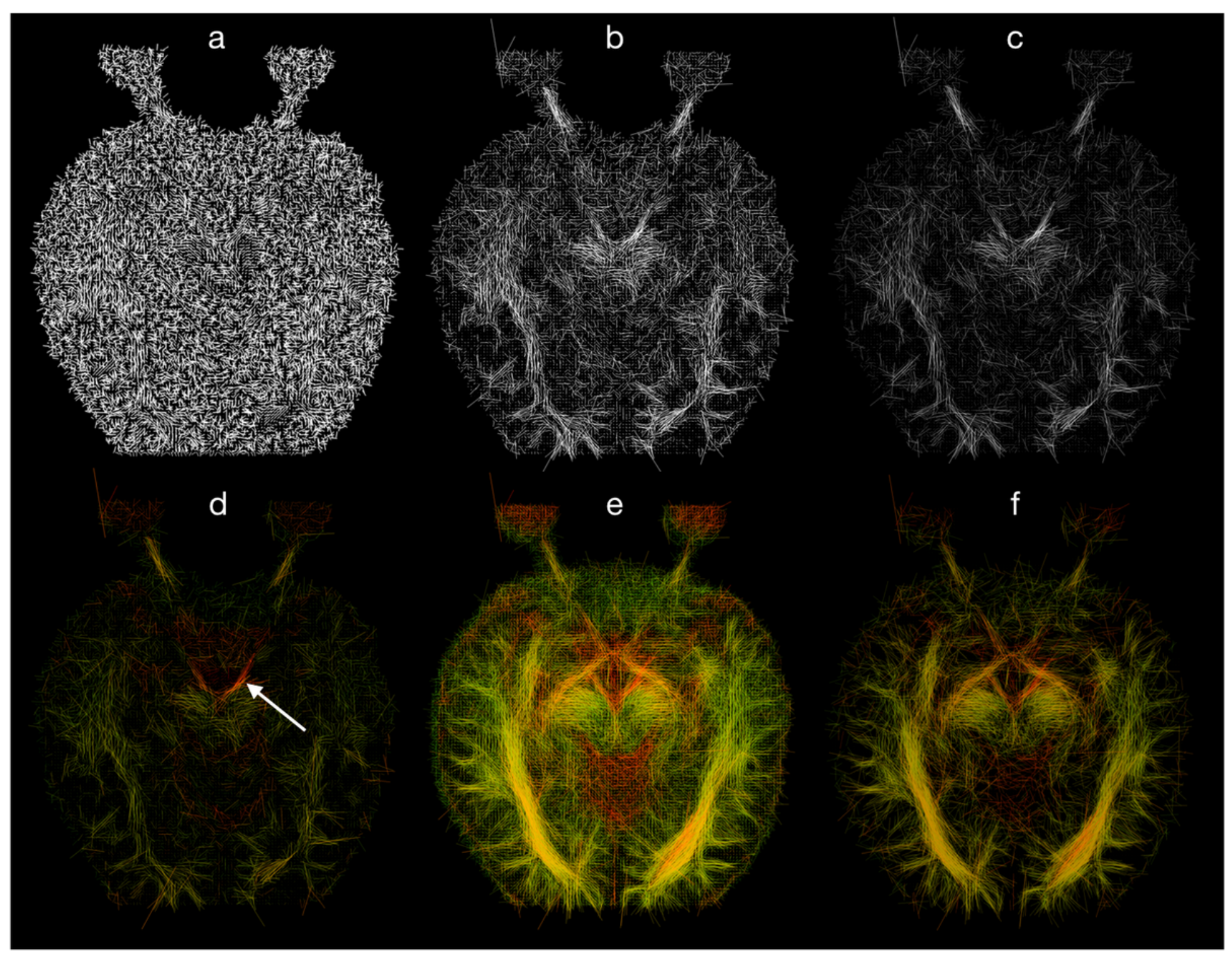

Figure 2

The visual effect of the algorithm in six steps for a healthy subject, a - principal diffusion directions shown as line segments, $b$ - length coding, $c$ - opacity coding, $d$ - color coding, e - seven layers projection, $\mathrm{f}$ - seven layers projection and data thresholding. In a-d a single layer was used. White arrow: oculomotor nerve. Orientation convention: Radiological. 


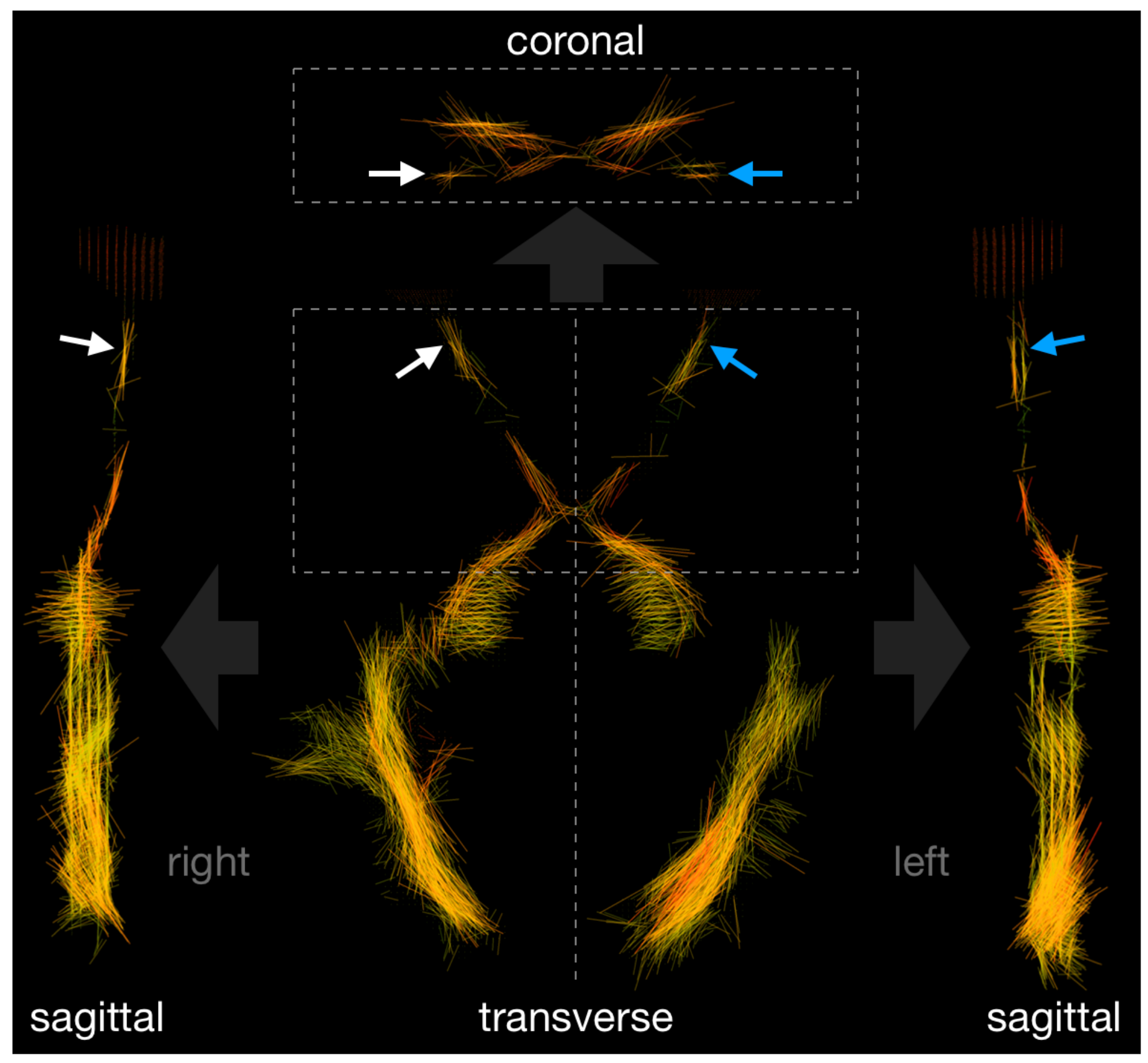

Figure 3

Visual pathway shape on different plane projections. White and blue arrows indicate right and left optic nerve, respectively. Real voxel proportions are preserved. 
a

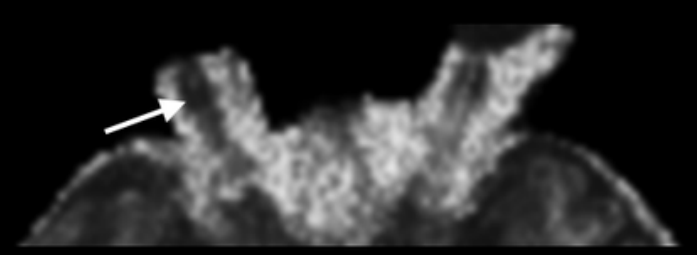

FA
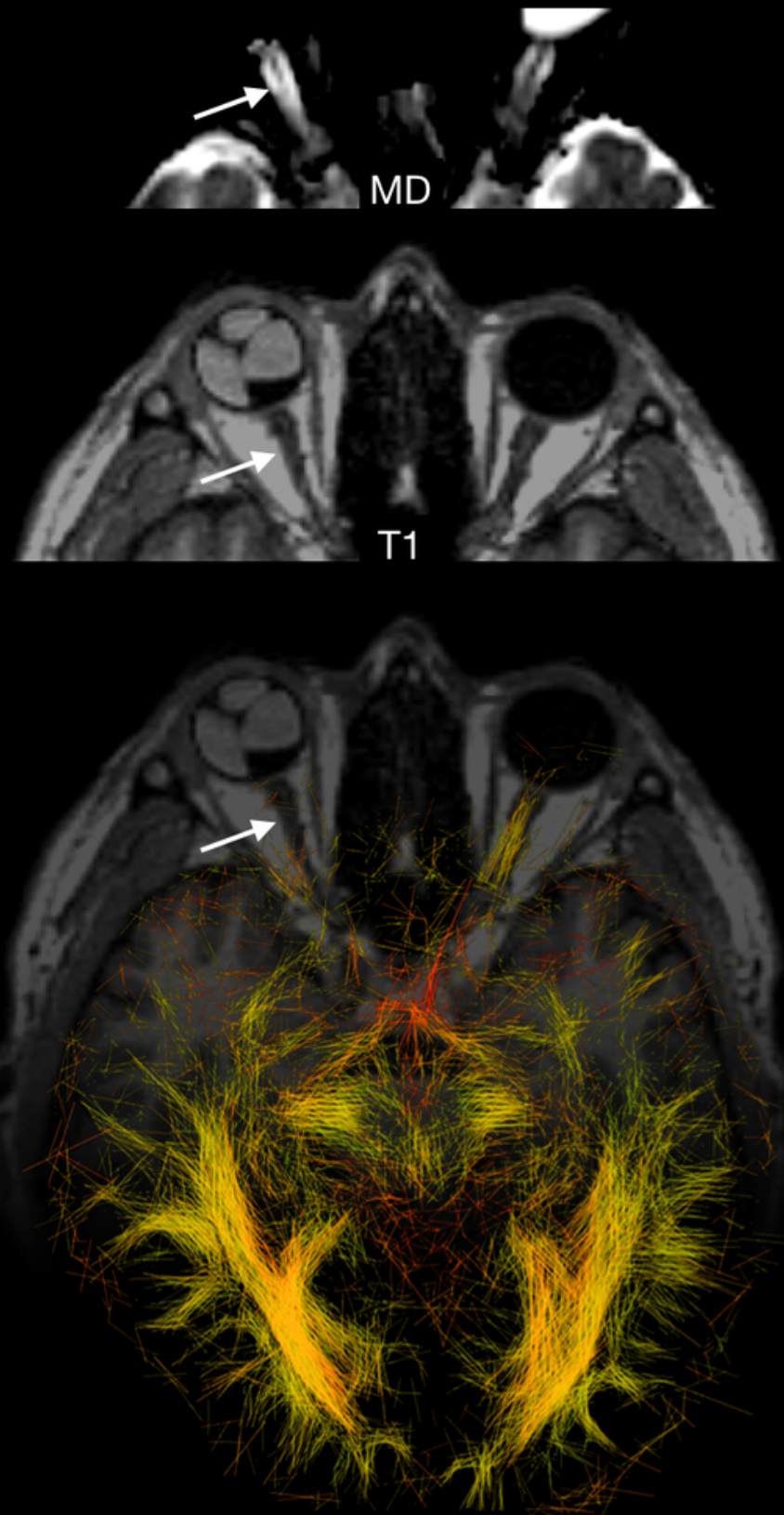

DTI and T1

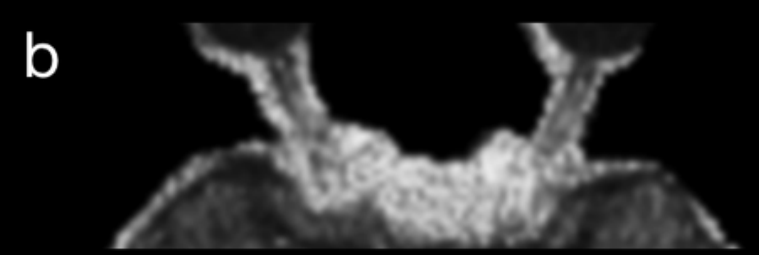

FA
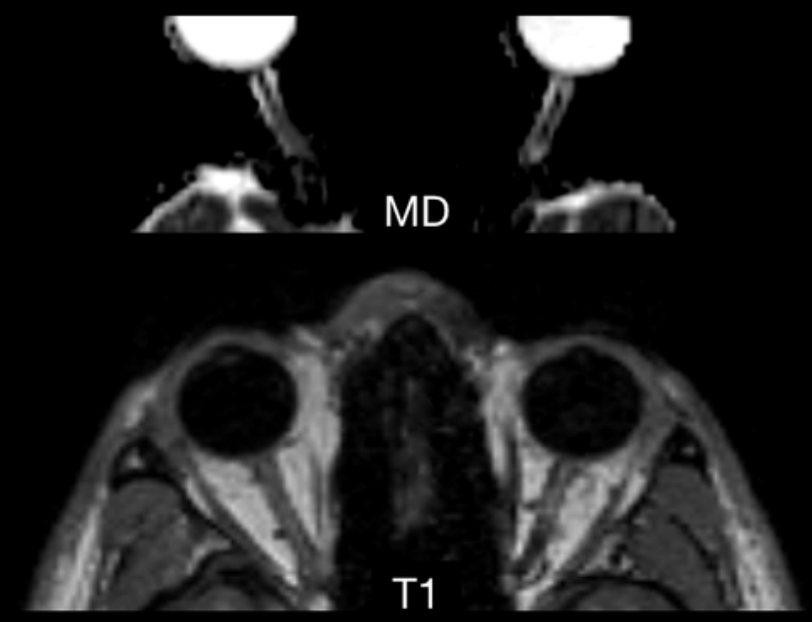

$\mathrm{T} 1$

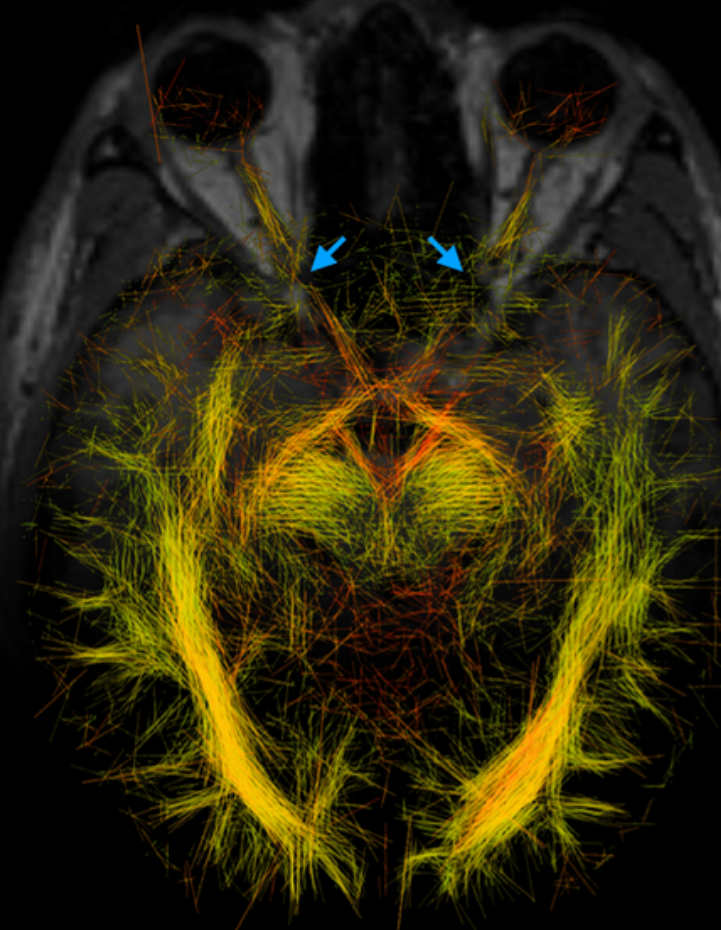

DTI and T1

\section{Figure 4}

Visual pathway of male subject with right optic nerve atrophy (white arrows) (a) and for healthy male subject (b) visualized with the new DTI algorithm superimposed on the same T1 image. Optic nerves are also presented on diffusion scalar maps FA and MD, and on morphological T1-weighted images. Blue arrows: optic channel and locations of visible discontinuities. Orientation convention: radiological. Values of $a, \beta, y$ and $\sigma$ used in visualization were $3.410^{7}, 500,2$ and $710^{-4}$, respectively. 


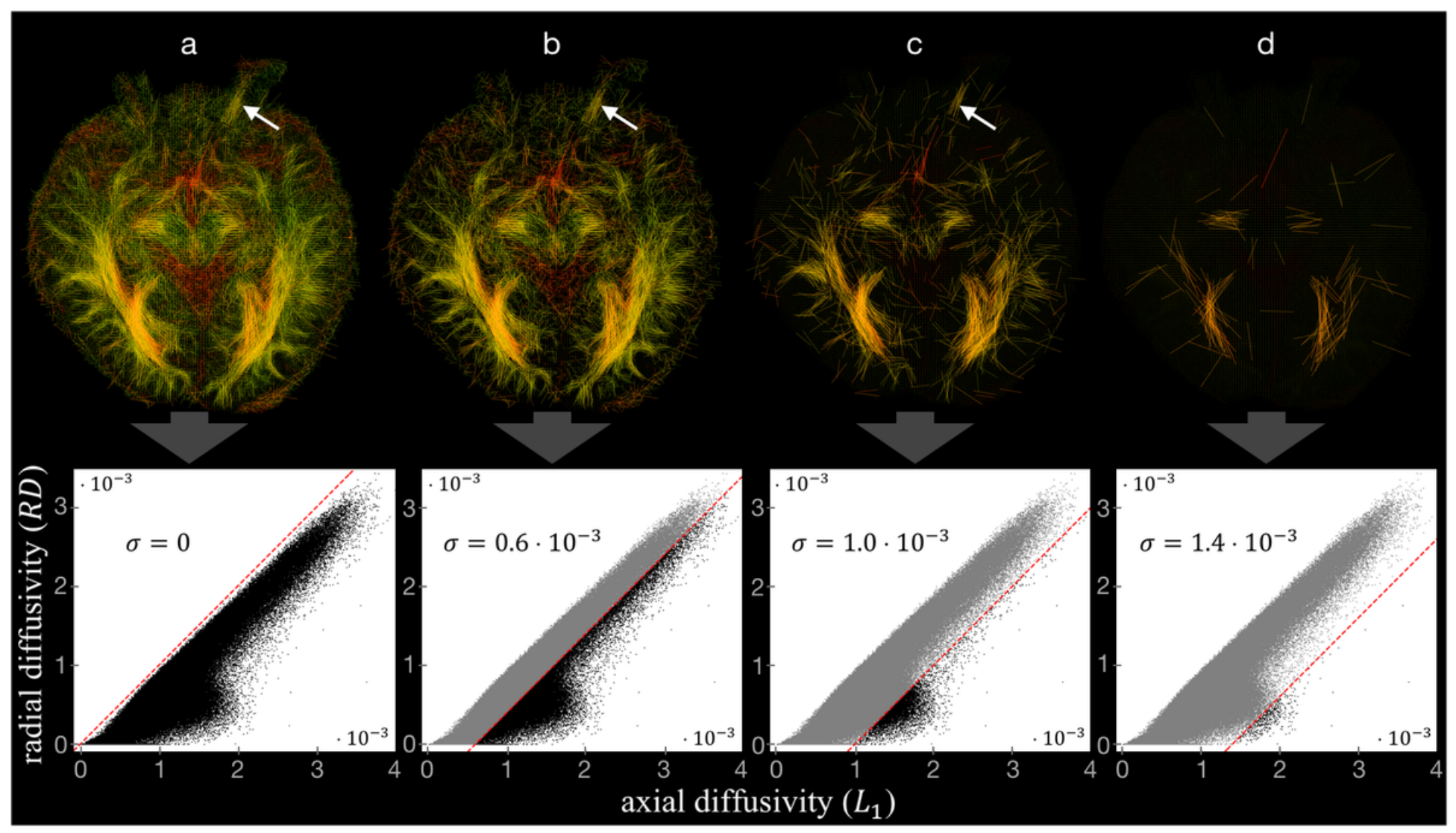

\section{Figure 5}

Images for male subject with right optic nerve neuropathy, generated for values equal to a) $0, b) 610^{-4}$, c) $1010^{-4}$, and d) $1410^{-4}$. Density plots in the bottom panels show voxels used for visualization (black dots) and voxels omitted according to condition (7) (grey dots). The red dashed lines correspond to the condition $L_{1}=R D+\sigma$. The left optic nerve (white arrow) is visible $(\mathrm{a}, \mathrm{b}, \mathrm{c})$ until a certain value of is exceeded (d). Orientation convention: Radiological. 